\title{
Research on the Artistic Language of Ceramic Painting in Jingdezhen Ceramic Decoration
}

\author{
Deming Long \\ Jingdezhen Ceramic Institute, Jingdezhen, Jiangxi 333403, China
}

\begin{abstract}
Keywords: Ceramic painting; Material; Arts and crafts; Arts language; Specificity; Aesthetic orientation
\end{abstract}

\begin{abstract}
Ceramic painting and other painting arts have obvious differences, it has its own artistic value. Ceramic painting has a strong craftsmanship, it is the crystallization of craft personality and painting universality, is the perfect combination of science and technology with arts. The uniqueness of ceramic painting materials and craftsmanship is the main factor that determines the particularity of ceramic art. Therefore, the ceramic painter should be on the basis of research of ceramic materials and technology, and then establish their own aesthetic orientation, in order to form a distinctively personal art language.
\end{abstract}

\section{Ceramic Painting Concept}

Ceramic painting is named by the painting carrier, that is, painting on the ceramic. Naming it by ceramic carrier show its characteristics, people remember it deeply. The artistic features of it have two distinctive features: firstly, it is ceramic, and the more typical feature is: it is porcelain painting. Secondly, it has the common cultural characteristics with variety of paintings. In the ceramic art, it can be with ceramic decoration, pottery together to constitute the three pillars of the Chinese contemporary ceramic art culture pattern. Ceramic painting and modern pottery are essentially different, modern pottery is the art category obviously influenced by foreign culture and independently developed.

Ceramic painting refers to using ceramic painting tools and ceramic materials to carry out artistic painting on two flat porcelain plates or on three-dimensional three-dimensional ceramic utensils. Ceramic painting was gradually developed originally drawn on the traditional ceramic decoration techniques and learning from Chinese painting, especially on the basis of literati painting. Therefore, it has a strong ceramic technology, but also a simple painting, it is crystallization of the process characteristics and painting universality, is the perfect combination of art and science and technology. In the ceramic painting, the artist's main consciousness can be fully developed and publicity, the emotional component of artistic expression is greater than the rational component.

\section{Research on Artistic Language of Ceramic Painting}

Every kind of art has its own artistic language, arts have significant difference because of its own unique artistic language, like that the country and even the nation with their own distinctive language, language and culture will show the cultural differences and characteristics. The art of ceramic painting has its own particularity and uniqueness. Because of this, it can serve as a kind of art independently standing in the field of artistic community. It shows its potential artistic vitality by its unique superiority.

The Factors Determining the Ceramic Art of Painting Their Own Specificity. Material factors. We know that porcelain is developed from pottery. Porcelain surface is usually attached to a layer of glaze, "glaze" is a silicate, after the calcination, it formed a layer of bright side. Hard texture and glazed exquisite are the basic characteristics for most porcelains, porcelain is the unity of porcelain and glaze. Adding some kind of oxidation of metal in the glaze, after the calcination, it will show a certain color. Carrying out painting performance on the special carrier of ceramics, not only the performance method and the obtained effect are different from the picture and the paper painting, it is worth studying and attention is that the effect of carrier material of the ceramic 
painting on its artistic language can not be ignored. Any painting art, the material of its painting carrier will be more or less acting on their artistic language, these carriers are generally through their own attributes to affect the performance techniques, and ultimately affect the overall art form and the formation of the special language of art. Ceramic material properties will also indirectly affect the performance and artistic effects of ceramic painting techniques, but in particular, the ceramic material with glaze is bright and clean, and the characteristics of ceramic paint, playing a great role in ceramic painting art language itself. These features are unmatched by other materials.

From the view of the use of the pigment in ceramic paintings, the rich variety and the beautiful texture are its salient features, which have the glaze color pigments rich in color with the outstanding performance - pastels, color, new color, but also have high temperature underglaze series, blue and white, colorful and color glaze. Here, this author take pastry and new color as an example, briefly summarize their pigment characteristics.

Pastel pigments with other pigments can not follow the advantages and characteristics, but also make the pink painting with unique characteristics. As the pastel color texture have the distinguish of transparent and opaque, but the gloss is very strong, all have the sense of powder, so the color material usually formed both uniform and change in the artistic effect. A rich color pink porcelain painting, with the function of light, the transparent painting with a certain sense of the thickness will appear to be bright, flourishing and elegant. Fluorescent pigments are equally bright. Transparency, opacity and glossiness are the inherent qualities of pastel colors, which make the art of teaser painting unique.

New color is also an important material applied to ceramic painting. It wins by its own performance. New color material can not only show the effect of Chinese painting, but also be similar to the effect of oil painting. New color paint have rich color, there is a certain degree of coverage, because before the burning, the pigment color is not changed, so easy to master. But fully grasping the unique nature of the new color pigment is not easy, only have a deep understanding on the characteristics of the pigment and butterfly oil, camphor oil and other blending oil, glue the characteristics, in order to fully show the unique performance of the new color paint. The new color pigments do not have the color of the pastel, but it also has a dazzling light character.

Process factors. Material factors and technological factors are the distinguishing features of ceramic painting, which is the main factor to determine the particularity of ceramic art. If the characteristics of ceramic materials constitute the most distinctive characteristics of ceramic painting, ceramic art used in the creation of the means of technology is displayed by the characteristics of ceramic materials. Talking about the process, we can not avoid the need to connect the painting material, because almost all of the material and craft techniques are closely related, ceramic painting is no exception, its technology is determined by the ceramic material, the unique properties of ceramic materials make techniques have their own characteristics, so as to further make the ceramic painting process to show difference in the art form and art language.

Craft technology in the ceramic painter seems both to express subjective feelings and the way to express self-awareness, but also used to reflect the charm of the material. The world of ceramic materials has a very broad unknown area, all the properties of ceramic materials, and the technology of using materials, are from unknown to the cognitive process, the material itself has a huge potential to be explored. Compared with painter's artistic thought, cultural quality, personality, temperament, knowledge, technology is in the second position, but the process technology is also an important part can't be ignored. Ceramic painting is also like this. Because the characteristics of ceramic painting material need to be fully reflected, only through a complete process techniques. New color paint is a considerable material with potential for the performance, to highlight the advantages, characteristics of this material, must continue to tap out with the appropriate technology. The technology derivative and the new ceramic painting art are continually expanded, art style diversification, so the art of ceramic painting language will be able to expand.

The Relationship between Ceramic Materials, Technology and Artistic Performance. Materials Determine the Process, While the Process Shows Materials. The relationship between material and process technology, in fact, is the relationship of the dynamic people, comply with the 
natural properties of the material to and grasp them. In the process, the compliance of the natural laws of the material is not passive, but active, is different nature and characteristics of the domination and compliance of the binding material, often determine the different types of goods and the corresponding technical attributes. The performance of the material comes from the internal properties of the material, the process is the process of processing methods and art processing methods by getting development based on the characteristics of various materials. Technology and materials complement each other, are closely related. Material, is the material of the process, the process is the disposal process of materials in the process. Materials is different from heterogeneous by their own different quality, technology is art of showing the characteristics.

Ceramic materials is very rich, making the ceramic painting technology rich and varied. There are not only the variety of ceramic pigments, and each has a distinctive feature and characteristics, ceramic painting technology have their own characteristics due to this reason. Ceramic materials with their own different quality is different from other works of art materials and shows their own process characteristics. It can be said that the porcelain painting process, to some extent, is to show the characteristics of ceramic art. Therefore, the color of the pastel water color and its own characteristics are reflected by the flat filling process. The process not only reflects the material properties, but also a slight change in the process will affect the artistic effect of the material presented. So the material and the process has a very delicate relationship, the material determines the process, and process shows materials.

With the development of ceramic art, with the growing of ceramic painting team, materials can be applied to the ceramic painting are more and more rich, different performance materials will derive different technology, in this derivative process, artists continue to understand the characteristics of materials, rationally using them, in order to create the necessary techniques of the artistic creation.

Processing Techniques Serve for the Performance of the Arts. Ceramic materials and technology are the main factors to determine the specific characteristics of the ceramic art, but not the ultimate goal in the pursuit of ceramic painting. In ceramic painting, ceramic materials and processes are serving for the performance of the arts. Ceramic painting are same to other types of painting, are pursuit the perfect unity of content and form, the fundamental pursuit of the purpose for them are based on the aesthetic value of the screen and spiritual content.

Ceramic carrier and ceramic pigment are the important media for ceramic painting, but not art. Ceramic painting material is special, unique craftsmanship, but the ceramic painting materials and processes are for the service of artistic expression. Studying the techniques of ceramic painting is the necessary way to master the language of ceramic painting, and serving for the creation of passionate works of art. If you leave the performance of ceramic art and the form of content, and then superb technology is only skill, not art. Superb technology, superb techniques, with the artistic appeal, and only embodied through the new art form, rich spiritual content.

Skills and painter's ideas, accomplishment, personality, knowledge are located in the secondary position, but the skill of using creative art is very important. On the other hand, we must pay attention to the visual appeal of ceramic painting art, we must emphasize the aesthetic value of ceramic painting and its artistry. On the other hand, we must pay attention to the visual appeal of ceramic painting art. Therefore, the ultimate goal of continuously improving the technology is to serve the art of painting, art technology is to serve the artistic expression. In the accepted technology, at the same time, we can not only rely on technical theory, for any one painting, technology is the means to complete the work, and the spirit of the screen is the soul of the work. Thus, the spiritual connotation of aesthetic characteristics for the ultimate connotation of the works to convey, ideological and emotional can give the works of true appeal and vitality. Ceramic painting as a picture, should also abide by the commonality of painting.

Artistic expression is the trajectory of artistic creation, the material manifestation of the spiritual labor of art, the artistic conception of all artistic ideas, only through the artistic image, can show the concrete, stable and sensed by the artistic expression of certain material forms. Artistic expression is materialization process through the combination of material content and form. The level of 
paintings can only be confirmed by the performance of the painting. So the painter must be dedicated to mobilize all the artistic skills and material means they can master, and strive to achieve the high degree of unity in content and form, technology and art.

\section{Acknowledgement}

Fund Project: Jiangxi Provincial Arts and Cultural Science Planning Project "Study on Ceramic Painting Art Language System Born out of Jingdezhen ceramic decoration" (Project Number: YG2016017)

\section{References}

[1] Y.Z. Li. Introduction to Arts and Crafts. Shandong Education Press.

[2] J.S. Wang. Chinese Painting Theory. Hunan Fine Arts Publishing House. 1999.8.

[3] B.L. Yuan. Comparative Art Course. Higher Education Press. 